\title{
Identification of Tumor-Associated Proteins in Well Differentiated Laryngeal Squamous Cell Carcinoma by Proteomics
}

\author{
Jian-rong Zhou • Zhong-xue Fu • Jie Li • \\ Lian-zhi Wei • Jun-cai Li
}

Published online: 17 June 2008

(C) Humana Press 2008

\begin{abstract}
Introduction This study established two-dimensional gel electrophoresis (2-DE) profiles for human well-differentiated laryngeal squamous cell carcinoma tissue and paired normal mucosa epithelia tissue and identified proteins with different expressions. Well-resolved and reproducible 2-DE patterns of well-differentiated laryngeal squamous cell carcinoma and adjacent normal mucosa were obtained.

Results Thirteen proteins were preliminarily identified, among which ten proteins including cofilin-1, nuclear body protein SP140, GRP94, HSP 90, GSTP1-1, superoxide dismutase $[\mathrm{Mn}]$, cyclophilin $\mathrm{A}$, proteasome activator complex subunit 2, apolipoprotein A-I precursor, and CaM-like protein were upregulated and three proteins including fatty acid-binding protein (E-FABP), calgranulin $\mathrm{A}$, and calgranulin $\mathrm{B}$ were downregulated in laryngeal cancer tissue. The different expressions of cyclophilin A and MRP8 were confirmed by Western blotting.

Discussion We first identified 13 proteins that might be associated with the tumorigenesis of the laryngeal squamous cell carcinoma. Some proteins were the products of oncogenes and apoptosis and others were related to signal transduction and immune defense. These extensive protein variations indicated that multiple protein molecules were simultaneously involved in the oncogenesis of laryngeal
\end{abstract}

J.-r. Zhou $\cdot$ J. Li $\cdot$ L.-z. Wei

Department of Otorhinolaryngology,

The First Affiliated Hospital of Chonging Medical University, Chongqing 400016, China

Z.-x. Fu $(\bowtie) \cdot$ J.-c. Li

Department of Surgery,

The First Affiliated Hospital of Chonging Medical University, Chongqing 400016, China

e-mail: fzx990521@sina.com cancer, which in turn is a basis for the rational designs of diagnostic and therapeutic methods.

Keywords Laryngeal neoplasms $\cdot$ Squamous cell carcinoma Two-dimensional gel electrophoresis (2-DE) . Matrix-assisted laser desorption/ionization time-of-flight mass spectrometry (MALDI-TOF-MS) · Proteomics

\section{Introduction}

Laryngeal cancers represent a major health problem worldwide, and laryngeal squamous cell carcinoma (LSCC) is the most common type of laryngeal cancer. It is the one not showing an increase in the 5-year survival rates over the last 30 years. The increasing use of chemo- and radiotherapy and conservative surgery to preserve organs and their functions has probably led to a better quality of life in patients with laryngeal cancer, but has definitely failed to improve survival, which remains the primary aim. This highlights the necessity for continued efforts to discover suitable biomarkers for early diagnosis of the disease and to understand its pathogenesis as a first step in improving methods of treatment. Proteomics provides an effective approach to study disease pathogenesis by globally examining the different protein expressions because of malignant cell transformation in disease $[1,2]$.We have recently identified tumor-associated proteins in LSCC by means of proteomic technology. These tumor-associated proteins can be further evaluated as potential biomarkers for clinical diagnosis and as targeted proteins for pathogenetic investigations. The present findings may shed light on the molecular characterization of laryngeal cancer progression and may be informative for identifying biomarkers and therapeutic targets for LSCC. 


\section{Materials and Methods}

\section{Tissue Specimens}

Tissues used in this experiment were obtained with the approval of the Committees for Ethical Review of Research involving Human Subjects at Chongqing Medical University. A total of eight human LSCC tissues from patients were collected immediately after isolation of surgically resected in the First Affiliated Hospital of Chong Qing Medical University, The eight cases included eight men with an average age of 57 years. Tissue samples were snapfrozen in liquid nitrogen and then preserved in $-80^{\circ} \mathrm{C}$ deep freezer or on dry ice for transfer before experiments. The histology for all the eight samples was confirmed by two independent histopathologist after fixation, embedding, sectioning, and hematoxylin-and-eosin staining. All samples comprised more than $80 \%$ of the targeted cells (normal epithelial cells or cancer cells) without necrosis. The American Joint Committee on Cancer pathologic stages were $\mathrm{T}_{2-4} \mathrm{~N}_{0} \mathrm{M}_{0}$.

\section{Preparation of Tissue Protein Samples}

Fresh frozen laryngeal tissue samples (100-200 mg) were cut into small pieces, dissolved in lysis buffer at the ratio of 80 -mg tissue per 1,000- $\mu$ l lysis buffer $(8 \mathrm{~mol} / 1$ urea, $2 \mathrm{~mol} /$ 1 sulfourea, $4 \%$ amphion detergent, $65 \mathrm{mmol} / 1$ dithiothreitol, $0.2 \%$ ampholite, $10 \mathrm{ml} / 1$ protease inhibitor cocktail), and then homogenized for $5 \mathrm{~min}$ on ice with a minihomogenizer. The mixture was centrifuged at $4^{\circ} \mathrm{C}$ for $15 \mathrm{~min}$ to remove tissue and cell debris. The supernatant was taken as extracted proteins, and the protein concentration was determined by the bicinchoninic acid method. Aliquots of protein samples were kept in $-80^{\circ} \mathrm{C}$ deep-freezer until further use.

\section{2-DE,Coomassie Staining, and Image Analysis}

Two-dimensional gel electrophoresis (2-DE) was carried out with Amersham Biosystems IPGphor IEF and Bio-Rad protein $\alpha$ XI cell vertical electrophoresis bath system (BioRad CO.) according to the protocol described [3]. All samples were run at least in duplicate to guarantee reproducibility. Two good quality gels for each case were included into the subsequent image analysis. Coomassie brilliant blue staining was performed as described [4].

Image Analysis and MS Peptide Sequencing

The image of two-dimensional gels were digitalized with an ImageScanner and were conducted with ImageMaster 2D v2002.1 software (Amersham Pharmacia). The normalized value for each protein spot volume was used for comparison. Only those spots that have statistical significance in differential expression were selected for further investigation. Protein spots were cut out of gels in small pieces and subjected to in-gel tryptic digestion overnight. Peptide mass spectra were recorded, and parameters for spectra acquisition were used as stated [3]. A Voyage-DE STR matrix-assisted laser desorption/ionization time-of-flight (MALDI-TOF) mass spectrometer (Applied Biosystems, Foster City, CA, USA) was employed to obtain the peptide mass spectra with the following settings: reflector mode with 175 ns delay extraction time, 60-65\% grid voltage, and $20 \mathrm{kV}$ accelerating voltage. Laser shots of 250 per spectrum were used to acquire the spectra with a mass range of 500-4,000 Da. Mass calibration was performed using autolytic fragment peaks of trypsin including 906.5049, 1,153.5741, and 2,163.0570 Da. Proteins were identified by peptide fingerprinting using MS-Fit to search the NCBInr protein database (http://prospector.ucsf.edu). In database protein matching using MS-Fit (http://prospector. ucsf.edu/), $25 \mathrm{ppm}$ or better mass accuracy and Mowse scores more than 200 were obtained in most of the analyses. Duplicate or triplicate runs were performed to ensure an accurate analysis. Western blotting was performed to confirm the specificity of the identified proteins, if necessary.

\section{Western Blotting}

Protein samples were run on gel using the identical conditions as stated for 2-DE in "2-DE, Coomassie Staining, and Image Analysis." The separated proteins in the corresponding areas from the gels were transferred onto polyvinylidene difluoride membranes and incubated overnight at $4{ }^{\circ} \mathrm{C}$ with a blocking buffer containing Tris-buffered saline, $0.1 \%$ Tween 20 (TBST), and 5\% nonfat dry milk. Membranes were washed with TBST and incubated with monoclonal or polyclonal antibodies at a 1:500 or $1: 1,000$ dilution for $1 \mathrm{~h}$ at room temperature or overnight at $4{ }^{\circ} \mathrm{C}$. After washing again with TBST, the membranes were blotted with a secondary antibody conjugated with horseradish peroxidase at a 1:10,000 dilution for $1 \mathrm{~h}$ and then detected by enhanced chemiluminescence (Santa Cruz Biotechnology, Inc., USA) for $1 \mathrm{~min}$.

\section{Results}

Protein Separation

Figure 1 is a typical two-dimensional gel image for the tumor tissue of LSCC. More than 800 protein spots were separated in a $13 \times 16-\mathrm{cm}$ gel, with magnetic resonance $(\mathrm{Mr})$ 


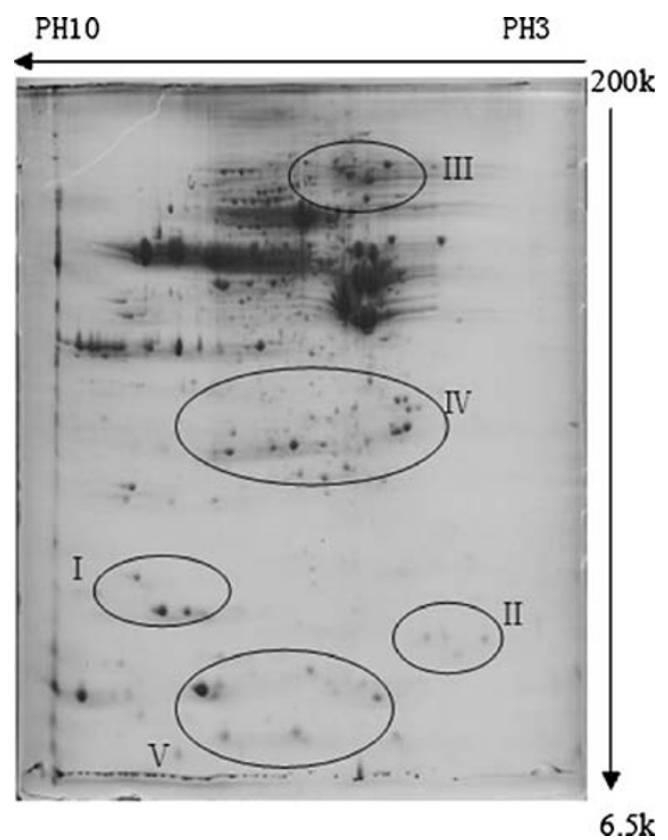

Fig. 1 2-DE image of LSCC

ranging from 6.5 to $200 \mathrm{kDa}$ and $\mathrm{p} I$ from 3 to 10 . Highlighted in squares are the five areas where significant and consistent volume differences of protein spots were found when comparisons were made between normal and tumor tissue samples. Except for three proteins (fatty acidbinding protein [FABP], calgranulin A, and calgranulin B) that were almost below the level of detection in tumor tissue, all other identified proteins were substantially upregulated in LSCC samples. Statistic data for these proteins are summarized in Table 1 with fold differences between tumor and normal mucosae samples (for a total of eight pairs indicated). Roughly ten proteins are upregulated, and three proteins are suppressed significantly in tumor tissues.
Protein Identification

Each protein spot was excised and subjected to in-gel tryptic digestion, MALDI-TOF mass measurement, and database matching. Isoforms that gave an identical primary structure in the protein matching were classified as one protein. Table 1 is a summary of the proteins identified. Figures 1 and 2 show the alterations in the expression levels of the identified proteins between tumor and normal mucosae. Proteins that consistently displayed an apparent increase in their expression level in tumor samples include cofilin-1, GRP94, HSP 90, GSTP1-1, superoxide dismutase [Mn], cyclophilin A, and calmodulin (CaM)-like protein. Proteins considerably suppressed in tumor samples included E-FABP, calgranulin A, and calgranulin B. The majority of these proteins have Mrs that match their experimental values determined from gels.

\section{Protein ID and Expression Confirmation by Western Blotting}

Western blotting was performed to verify two selected proteins, cyclophilin A and calgranulin A, that may play functional roles in tumorigenesis. Figures 3 and 4 shows the Western blotting results for the two proteins. Figure 4 displays the representative gels of Western blotting, confirming the decreased expressions of calgranulin A in tumor tissues. Western blotting results shown in Fig. 3 exhibit the increased expression levels of cyclophilin A.

\section{Discussion}

Although numerous studies in the genomic field have revealed a magnitude of changes occurring in the multi-

Table 1 Statistic data for these proteins with fold differences between tumor and normal mucosae samples

\begin{tabular}{|c|c|c|c|c|c|c|c|}
\hline Spot & Protein name & Area & $\mathrm{p} I$ & Peptides matched & Molecular mass & Mowse score & Change (fold) \\
\hline 1 & Cofilin-1 & I & 8.3 & 5 & 18,401 & 3,547 & -2.7 \\
\hline 2 & Cyclophilin A & I & 7.8 & 8 & 17,881 & $7.11 \mathrm{e}+6$ & -6.6 \\
\hline 3 & CaM-like protein & II & 4.3 & 10 & 16,760 & 77,670 & -1.5 \\
\hline 4 & GRP94 & III & 4.8 & 13 & 92,470 & $2.17 \mathrm{e}+9$ & -3.9 \\
\hline 5 & HSP90 & III & 5.0 & 17 & 83,134 & $4.94 \mathrm{e}+10$ & -4.2 \\
\hline 6 & Nuclear body protein SP140 & III & 5.3 & 8 & 99,871 & 35,504 & -3.3 \\
\hline 7 & Glutathione S-transferase P & IV & 5.4 & 11 & 23,225 & $5.26 \mathrm{e}+8$ & -1.4 \\
\hline 8 & Superoxide dismutase [Mn] & IV & 6.9 & 7 & 23,673 & 1,042 & -1.8 \\
\hline 9 & Proteasome activator complex subunit 2 & IV & 5.4 & 6 & 27,231 & 192,232 & -3.6 \\
\hline 10 & Apolipoprotein A-I precursor & IV & 5.7 & 18 & 30,277 & $7.85 \mathrm{e}+10$ & -4.0 \\
\hline 11 & Fatty acid-binding protein, epidermal & $\mathrm{V}$ & 6.8 & 9 & 15,033 & $1.70 \mathrm{e}+6$ & +2.9 \\
\hline 12 & Calgranulin B & $\mathrm{V}$ & 5.7 & 4 & 13,242 & 6,625 & +3.4 \\
\hline 13 & Calgranulin A & $\mathrm{V}$ & 6.5 & 6 & 10,835 & 29,279 & +5.2 \\
\hline
\end{tabular}

1-10 the protein spots upregulate, $11-13$ the protein spots downregulate 


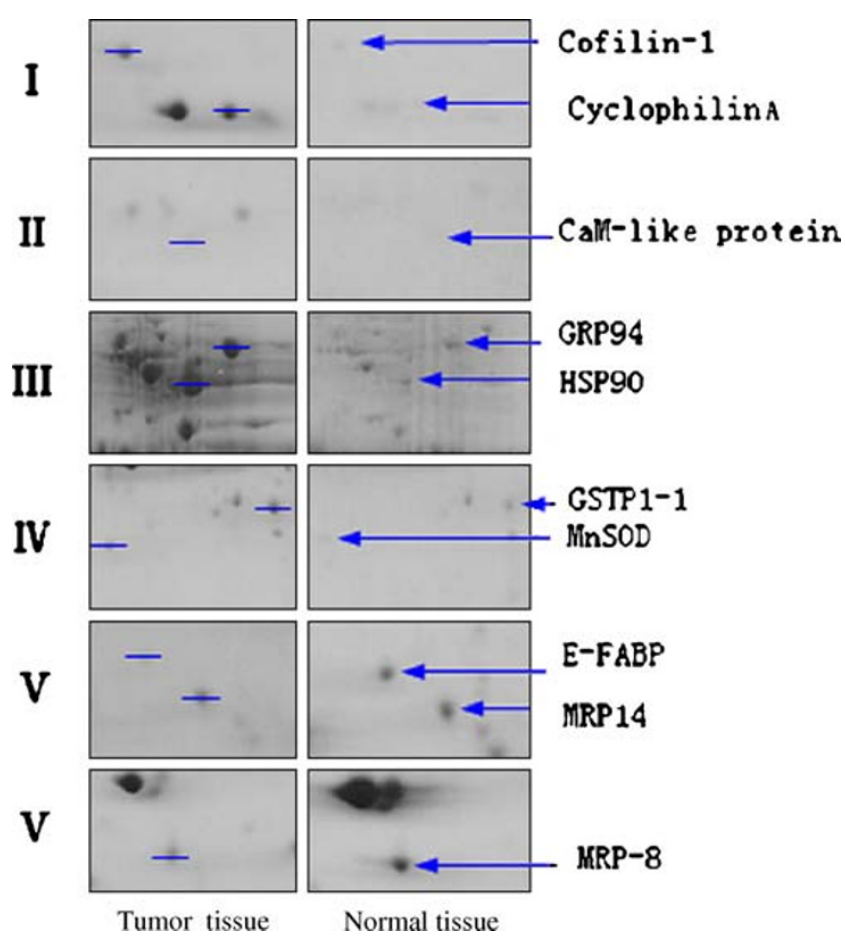

Fig. 2 Protein alterations in areas $\mathrm{I}-\mathrm{V}$

stage pathogenesis of LSCC, including mutations of a variety of tumor suppressor genes and oncogenes, changes in transcription, proliferation-associated factors, and metastasis-related factors, these changes may not necessarily warrant subsequent corresponding alternations at the protein levels or functions. Proteomics, which aims at characterizing the entire protein complement expressed in cells or tissues, provides complementary information and direct evidence to unravel tumor-specific molecular events during multistage carcinogenesis. In the present study, we used 2-DE-based proteomics to examine the protein profiles of cancer tissues and the adjacent nontumor tissues and to identify proteins related to laryngeal malignancy. A total of 13 proteins were uncovered with differential expressions in laryngeal carcinogenesis, among which ten were upregu-

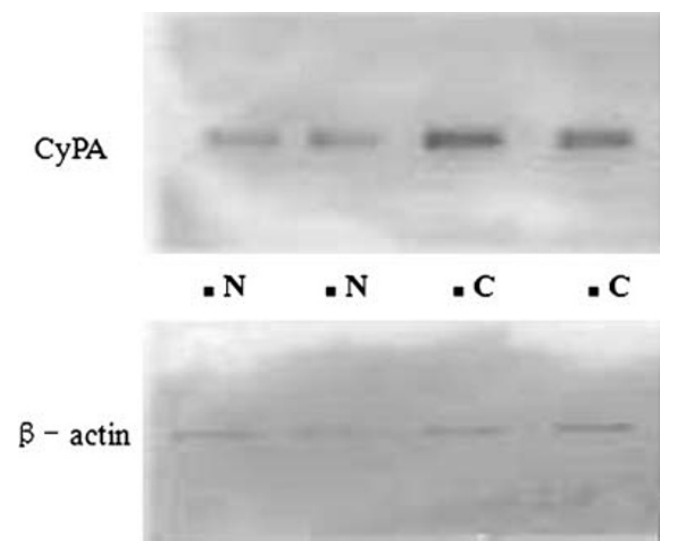

Fig. 3 Expression of CypA in laryngeal carcinoma and normal tissue

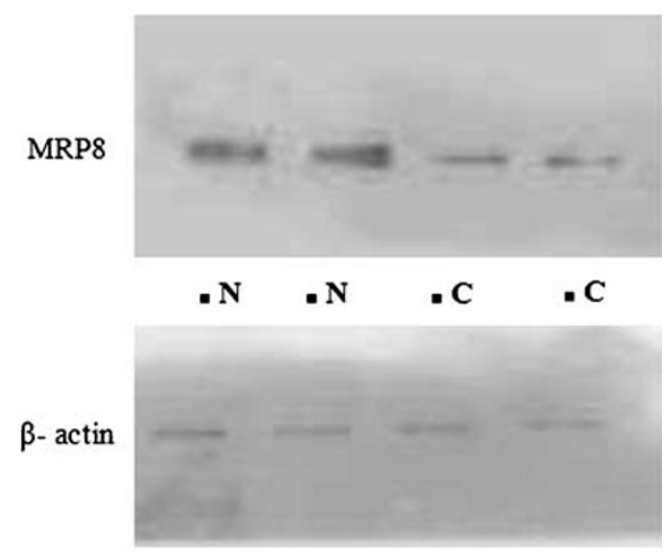

Fig. 4 Expression of MRP8 in laryngeal carcinoma and normal tissue

lated and three were downregulated. In summary, we used two-dimensional gel-based proteomics to compare the protein profiles between LSCC tumor and matched surrounding tissues and to identify differently expressed proteins in the laryngeal cancer. A number of tumorassociated proteins proved that proteomic analysis can provide rich information to help understand the pathology of a disease in an integrated way. This type of information should lead to more rationally designed diagnostic and treatment methods, which will hopefully translate into improved patient outcome.

CypA is a multifunctional cytokine involved in many biological processes, including protein folding, immunosuppression, cellular signaling, and apoptosis. Overexpression of CypA also was observed in non-small-cell lung cancer [5] and in human oral cancer cell lines [6] and pancreatic cancer. CypA could stimulate Panc-1 cell proliferation in a dose-dependent manner through its cellular receptor CD147 by activating the ERK1/2 and p38 pathways. Overexpression of CypA in laryngeal carcinoma indicated that it might play an important role in carcinogenesis, invasive action, and metastasis of LSCC.

Calgranulin A and calgranulin B also are named S100A8/MRP8 and S100A9/MRP14, respectively. S100 proteins, a multigenic family of calcium-binding proteins, have been linked to human pathologies in recent years. Deregulated expression of S100 proteins, including S100A8 and S100A9, was reported in association with neoplastic disorders. Aberrant S100A8 or S100A9 expression has been reported in a variety of epithelial tumors, including gastric, ovarian, colon, and breast cancers [7, 10] and prostate carcinomas $[8,11]$, as well as pulmonary and pancreatic adenocarcinomas [12, 13]. Interestingly, S100A8 is constitutively expressed in terminally differentiated squamous epithelium but lost in squamous cell carcinomas, such as esophageal squamous cell carcinoma, correlating with poor differentiation $[14,15]$. However, the detection of $\mathrm{S} 100 \mathrm{~A} 8 / 9$ can be used to discriminate pancreatic cancer 
from pancreatitis [13] and ovarian cancer from benign ovarian cysts [9] lending support to an overexpression in tumors. Nevertheless, our current study revealed that S100A8 and S100A9 were downregulations in the tissues of laryngocarcinoma. The pathologic significance of their discordant expression in different tumors will be our research topic in future.

Also known as ER modulin, GRP94 is a member of HSP90 family. HSP90 is usually considered as the cell survival factor, when the cell is stimulated by environment, will enhance expression of the heat shock protein. It can prevent the cell from attack of the endogenous stress quickly and transiently, reinforce the ability of recovery, and enhance the tolerance to the stress [16]. GRP94 plays an important role in the tumorigenesis and the survival of the tumor cells. As an important part of oncogene pathway, many signal transducers depend on HSP90 to play their role. In laryngeal carcinoma, GRP94 and HSP90 upexpressions indicate that the Ego defense mechanism of the cell is activated to cope with the malignant transformation of the cell.

$\mathrm{CaM}$ is one of the most important intracellular calciumbinding protein, and it is the chief intracellular $\mathrm{Ca}^{2+}$ signal transmitter. As a multifunctional enzyme regulator, $\mathrm{CaM}$ is concerned with multitude vital phenomena, it influences cell growth and cell proliferation, and also, it may play a role in regulating the oncogene protein [17]. Many researches have found that $\mathrm{CaM}$ has a higher level in tumor cells such as breast cancer, leukemia, hepatoma, and lung cancer. It was confirmed that $\mathrm{CaM}$ has inhibitory action on proliferation and metastasis of the tumor in years 1970-1980.

COF1 probably participates in cell movement through reversible phosphorylation regulation of actin and plays an important role in tumor metastasis [18]. Some researchers have pointed out that adenosine-triphosphate-supported actin filament treading pedal is the impetus basis of actin persistent movement and this effect is regulated by COF, microfilament binding protein profiling, and capping protein. The depolymerization and polymerization of the actin promote the movement and metastasis of tumor cells.

Manganese superoxide dismutase (MnSOD) and glutathione $\mathrm{S}$ transferring enzyme $\mathrm{P}$ are intracellular frequent antioxidase. Increase of MnSOD indicates the activation of the familiar antioxidize defense mechanism. Many researches showed that MnSOD can prevent the cell from damage and can inhibit tumor growth, probably through changing the oxidation-reduction reaction circumstances of chondriosome and metabolic capability of the cell [19]. GSTP1-1 belongs to the cytolist enzyme family, participating in the process of detoxication [20]. In many kinds of tumors such as didymus tumor, ovaries tumor and colon tumor, overexpression of GSTP1-1 is associated with tumorigenesis and tumor development.
E-FABP, a familiar FABP, is associated with the psoriasis, which is in high-level expression in epidermis of psoriasis [21]. Its high-level expression was also found in bladder, pancreatic, and head and neck squamous carcinomas. The overexpression of E-FABP was also correlated to tumor metastasis. In contrast, E-FABP was found in low-level expression in tissues of laryngocarcinoma compared with the normal tissues in this study; further analysis to understand this inconsistence is in progress.

In conclusion, a number of proteins were found to be significantly altered in well-differentiated laryngeal carcinoma tissues by comparing to the adjacent non-tumor tissues through proteomic analysis, suggesting that multiple molecular pathways were involved in the tumorigenesis. Some of the identified proteins were validated by Western blotting to confirm their altered expressions in tissues. These tumor-associated proteins can be further characterized and evaluated as potential biomarkers for diagnosis of laryngeal carcinoma or as target molecules for pathogenetic study.

Acknowledgments We thank members of the laboratory for discussion, advice, and critical reading of the manuscript. We also acknowledge Mr. Pujiang Dong for excellent technical assistance with 2-DE and Kaishun Huang for excellent cooperation with mass spectrometric identification of proteins.

\section{References}

1. Lee EY, Bang JY, Park GW, et al. Global proteomic profiling of native outer membrane vesicles derived from Escherichia coli. Proteomics 2007;7(17):3143-53.

2. Jagtap P, Michailidis G, Zielke R, et al. Early events of Bacillus anthracis germination identified by time-course quantitative proteomics. Proteomics 2006;6(19):5199-211.

3. He QY, Lau GK, Zhou Y, et al. Serum biomarkers of hepatitis B virus infected liver inflammation: a proteomic study. Proteomics 2003;3:666-74.

4. Tanaka N, Mitsui S, Nobori H, Yanagi K, Komatsu S. Expression and function of proteins during development of the basal region in rice seedlings. Mol Cell Proteomics 2005;4:796-808.

5. Campa MJ, Wang MZ, Howard B, Fitzgerald MC, Patz EF Jr. Protein expression profiling identifies macrophage migration inhibitory factor and cyclophilin a as potential molecular targets in non-small cell lung cancer. Cancer Res 2003;63:1652-6.

6. Rey O, Baluda MA, Park NH. Differential gene expression in neoplastic and human papillomavirus-immortalized oral keratinocytes. Oncogene 1999;18:827-31.

7. Seth A, Kitching R, Landberg G, Xu J, Zubovits J, Burger AM. Gene expression profiling of ductal carcinomas in situ and invasive breast tumors. Anticancer Res 2003;23:2043-51.

8. El-Rifai W, Moskaluk CA, Abdrabbo MK, Harper J, et al. Gastric cancers overexpress S100A calcium-binding proteins. Cancer Res 2002;62:6823-6.

9. Ott HW, Lindner H, Sarg B, Mueller-Holzner E, et al. Calgranulins in cystic fluid and serum from patients with ovarian carcinomas. Cancer Res 2003;63:7507-14. 
10. Chaurand P, DaGue BB, Pearsall RS, Threadgill DW, Caprioli RM. Profiling proteins from azoxymethane-induced colon tumors at the molecular level by matrix-assisted laser desorption/ ionization mass spectrometry. Proteomics 2001;1:1320-6.

11. Rehman I, Azzouzi AR, Catto JW, Allen S, et al. Proteomic analysis of voided urine after prostatic massage from patients with prostate cancer: a pilot study. Urology 2004;64:1238-43.

12. Arai K, Teratani T, Nozawa R, Yamada T. Immunohistochemical investigation of S100A9 expression in pulmonary adenocarcinoma: S100A9 expression is associated with tumor differentiation. Oncol Rep 2001;8:591-6.

13. Shen J, Person MD, Zhu J, Abruzzese JL, Li D. Protein expression profiles in pancreatic adenocarcinoma compared with normal pancreatic tissue and tissue affected by pancreatitis as detected by two-dimensional gel electrophoresis and mass spectrometry. Cancer Res 2004;64:9018-26.

14. Melle C, Ernst G, Schimmel B, Bleul A, et al. A technical triade for proteomic identification and characterization of cancer biomarkers. Cancer Res 2004;64:4099-104.

15. Kong JP, Ding F, Zhou CN, Wang XQ, et al. Loss of myeloid-related proteins 8 and myeloid-related proteins 14 expression in human esophageal squamous cell carcinoma correlates with poor differentiation. World J Gastroenterol 2004; 10:1093-7.

16. Bishop SC, Burlison JA, Blagg BS. Hsp90: a novel target for the disruption of multiple signaling cascades. Curr Cancer Drug Targets 2007;7(4):369-88.

17. Weitzman S. Complementary and alternative (CAM) dietary therapies for cancer. Pediatr Blood Cancer 2008;50(2 Suppl):494-7.

18. Ukitsu H, Kuromori $\mathrm{T}$, Toyooka $\mathrm{K}$, et al. Cytological and biochemical analysis of COF1, an Arabidopsis mutant of an ABC transporter gene. Plant Cell Physiol 2007;48(11):1524-33.

19. Oberley LW. Mechanism of the tumor suppressive effect of MnSOD overexpression. Biomed Pharmacother 2005;59(4): $143-8$.

20. Meiers I, Shanks JH, Bostwick DG. Glutathione S-transferase pi (GSTP1) hypermethylation in prostate cancer: review 2007. Pathology 2007;39(3):299-304.

21. Chmurzyńska A. The multigene family of fatty acid-binding proteins (FABPs): function, structure and polymorphism. J Appl Genet 2006;47(1):39-48. 